\title{
Multiple origins of asteroid pairs
}

\author{
Seth A. Jacobson ${ }^{1,2}$ \\ ${ }^{1}$ Laboratoire Lagrange, Observatoire de la Côte d'Azur, \\ Boulevard de l'Observatoire, CS 34229, F 06304 Nice Cedex 4, France \\ ${ }^{2}$ Bayerisches Geoinstitut, Universtät Bayreuth, \\ D 95440 Bayreuth, Germany \\ email: seth.jacobson@oca.eu
}

\begin{abstract}
Rotationally fissioned asteroids produce unbound asteroid pairs that have very similar heliocentric orbits. Backward integration of their current heliocentric orbits provides an age of closest proximity that can be used to date the rotational fission event. Most asteroid pairs follow a predicted theoretical relationship between the primary spin period and the mass ratio of the two pair members that is a direct consequence of the YORP-induced rotational fission hypothesis. If the progenitor asteroid has strength, asteroid pairs may have higher mass ratios or faster rotating primaries. However, the process of secondary fission leaves the originally predicted trend unaltered. We also describe the characteristics of pair members produced by four alternative routes from a rotational fission event to an asteroid pair. Unlike direct formation from the event itself, the age of closest proximity of these pairs cannot generally be used to date the rotational fission event since considerable time may have passed.
\end{abstract}

Keywords. minor planets, asteroids; planets and satellites, formation; planets and satellites, individual (3749 Balam, 8306 Shoko)

Asteroid pairs are a population of main belt asteroids that have nearly identical heliocentric orbits. These occur at a frequency in excess of that expected by random fluctuations of background asteroid orbit density (Vokrouhlický and Nesvorný 2008; Pravec and Vokrouhlický 2009). Furthermore, when the orbits of these asteroid pairs are carefully integrated backwards, many have close encounters in phase space (small distance and low velocity) in the recent past (Vokrouhlický and Nesvorný 2009). This suggested a common origin, and the YORP-induced rotational fission hypothesis was immediately proposed (Vokrouhlický and Nesvorný 2008). Matching spectral types between pair members complements a common origin hypothesis (Duddy et al. 2012; Moskovitz 2012; Wolters et al. 2014). If a common origin hypothesis is accepted than these pairs provide powerful tools to test many theories including space weathering, mutual body tides and binary evolution.

\section{YORP-induced rotational fission hypothesis}

The YORP-induced rotational fission hypothesis is a widely explored set of arguments that endeavors to explain the existence and properties of asteroid pairs, binaries and other multi-member systems from the rotational fission of rubble pile asteroids due to the rotational acceleration of the YORP effect (Rubincam 2000; Bottke et al. 2002; Scheeres 2007a; Ćuk 2007; Walsh et al. 2008; Pravec et al. 2010; Jacobson and Scheeres 2011a). The YORP effect is the spin and orbit averaged rotational torque on an asteroid due to thermal radiation (Rubincam 2000), and its effects have been directly observed in nature (Vokrouhlický et al. 2003; Taylor et al. 2007; Lowry et al. 2007; Kaasalainen et al. 2007). The theory for this effect is well-developed for principal axis rotating asteroids (e.g. 
Vokrouhlický and Čapek 2002; Breiter et al. 2007; Nesvorný and Vokrouhlický 2007; Scheeres 2007b), and the rotational acceleration is:

$$
\dot{\omega}_{Y}=\frac{3 Y}{4 \pi \rho R^{2}}\left(\frac{G_{1}}{a_{\odot}^{2} \sqrt{1-e_{\odot}^{2}}}\right)
$$

where $G_{1}=10^{14} \mathrm{~kg} \mathrm{~km} \mathrm{~s}^{-2}$ is the solar constant at an astronomical unit divided by the speed of light, $a_{\odot}$ and $e_{\odot}$ are the asteroid's heliocentric semi-major axis and eccentricity, respectively, $\rho$ and $R$ are the density and radius of the asteroid, respectively, and $Y$ is the YORP coefficient (Scheeres 2007b). The YORP coefficient is mostly dependent on the shape and orientation of the body but explicitly not size-dependent. Observed values of the YORP coefficient lay between $10^{-3}$ and $10^{-2}$ (Taylor et al. 2007; Kaasalainen et al. 2007). While the YORP effect is prevalent on all small bodies in the near-Earth region, the strong dependence on size and distance limits the consequences of this phenomena to small asteroids with radii $R \lesssim 6 \mathrm{~km}$ in the main asteroid belt (Jacobson et al. 2014a). However, there is a less-developed so-called tangential component that can increase the strength of the YORP torque for prograde rotators by a factor of two and so may extend this size domain by a few tens of percent; perhaps more importantly, this tangential component has a prograde bias (Golubov and Krugly 2012; Golubov et al. 2014).

For asteroids undergoing spin up due to the YORP effect, centrifugal accelerations increase throughout the body, but they are resisted by gravity, cohesive forces and mechanical strength. Centrifugal accelerations match gravitational accelerations for a planar two sphere approximation, when the spin rate $\omega$ reaches a critical value:

$$
\omega_{c}=\omega_{d} q_{d} \quad q_{d}=\sqrt{\frac{1-q^{1 / 3}+q^{2 / 3}}{\left(1+q^{1 / 3}\right)^{2}}}
$$

where $\omega_{d}=\sqrt{4 \pi G \rho / 3}$ is the critical disruption spin rate for a test particle on the surface of a constant density sphere, $G$ is the gravitational constant, $\rho$ is the density of the asteroid, $q_{d}$ is a function of the mass ratio $q$, which is the mass of the smaller, secondary component $m_{s}$ divided by the mass of the larger, primary component $m_{p}$ (Hartmann and Larson 1967). If the connection between the two components does not possess any strength, then when the spin rate reaches this critical value, the two components enter into orbit about each other.

If the internal structure of the asteroid has strength, then the asteroid goes into tension as the YORP effect increases the spin rate past the critical rate described above. For the planar two sphere approximation, the expression for the critical spin rate can be expressed simply as:

$$
\omega_{c}=\sqrt{\left(\omega_{d} q_{d}\right)^{2}+\left(\omega_{t} q_{t}\right)^{2}} \quad q_{t}=\sqrt{\frac{\left(1-q^{1 / 3}+q^{2 / 3}\right)(1+q)^{2 / 3}}{q^{1 / 3}}}
$$

where $\omega_{t}=\sqrt{3 \sigma_{c} /\left(4 \pi \rho R^{2}\right)}$ is a simple prescription for the critical rotation needed to disrupt a progenitor asteroid of effective radius $R$ with a critical strength $\sigma_{c}$ and no gravity (Sánchez and Scheeres 2014), and the factor $q_{t}$ scales the area of the stressed internal surface with the size of the smaller, secondary fissioned mass. Naturally, failure occurs along the weakest internal surface in the body and this determines the mass ratio $q$ of the orbiting components. Note, while this equation is dimensionally correct and acceptable for order of magnitude estimates, more detailed and accurate formulae are available that are derived directly from the application of the Drucker-Prager criterion on 
the von Mises stress and incorporate the role of cohesion as well (Holsapple 2001; Sharma 2009; Sánchez and Scheeres 2014). Furthermore, we note the similarity between our simple critical spin limit calculated above and those calculated by Sánchez and Scheeres (2014). They consider the role of cohesion as characterized by a material friction angle and find that variations of the friction angle from $0^{\circ}$ to $90^{\circ}$ produce only a factor of two change in the critical tensile strength spin limit.

There are only a few estimates of the critical strength of asteroids available. Examining the spin rate distribution of near-Earth and small main belt asteroids as a function of radius, there is a clear $\sim 2.3 \mathrm{hr}$ spin period limit for asteroids larger than $\sim 0.2 \mathrm{~km}$ (Harris 1996; Pravec and Harris 2000; Pravec et al. 2007); some smaller asteroids are observed to rotate much faster (Pravec and Harris 2000; Hergenrother and Whiteley 2011). This pattern and the lack of binaries with primary radii less than $200 \mathrm{~m}$ can be understood if a typical asteroid critical strength is less than $\sim 25-100 \mathrm{~Pa}$ (Sánchez and Scheeres 2014). The rapidly rotating asteroid 290751950 DA requires a lower limit on the critical strength of $\gtrsim 75 \mathrm{~Pa}$ (Rozitis et al. 2014; Hirabayashi and Scheeres 2015). The only direct estimate is derived from the disintegration of P/2013 R3 and suggests a critical strength of 40$210 \mathrm{~Pa}$ for this $165 \mathrm{~m}$ radius asteroid (Hirabayashi et al. 2014). For the ensuing simple calculations, an asteroid's critical strength is assumed to have an inverse dependence on the square root of the size of the body: $\sigma_{c}=\sigma_{c, 0} \sqrt{R_{0} / R}$ where $\sigma_{c, 0}=125 \mathrm{~Pa}$ and $R_{0}=165 \mathrm{~m}$. This relation follows the general understanding that mechanical strength is determined by flaws, which grow in size with the body, in analogy to Griffith's crack theory assuming a Weibull distribution of cracks.

\section{Free energy after a rotational fission event}

The critical spin rate determines the free energy of a newly formed binary system after a YORP-induced rotational fission event. The free energy is the sum of the rotational kinetic energy of each body, their relative translational kinetic energy, and their mutual gravitational potential energy. For two spheres in contact, which is the simplest approximation for a body undergoing rotational fission at the moment of fission (Scheeres 2007a), the initial free energy $E_{i}$ of a rotationally fissioned binary is:

$$
\frac{E_{i}}{E_{c}}=q_{1} q_{d}^{2}-q_{2} \quad q_{1}=\frac{2+q\left(7+q^{\frac{1}{3}}\left(10+q^{\frac{1}{3}}(7+2 q)\right)\right)}{q(1+q)^{\frac{2}{3}}} \quad q_{2}=\frac{10(1+q)^{\frac{1}{3}}}{1+q^{\frac{1}{3}}}
$$

where $E_{c}=2 \pi \rho q \omega_{d}^{2} R^{5} / 15(1+q)^{2}$ is an energy normalization constant (Scheeres 2009).

This free energy is the energy accessible to each of the energy reservoirs: the spin of each body and their relative motion. The spin-orbit coupling of the higher-order nonKeplerian gravitational potentials of the two components with the mutual orbit (and, to a lesser extent, tides) transfers energy between these reservoirs. If the energy in the orbit becomes positive then the system is on a disruption trajectory and will become unbound once the two components reach their mutual Hill sphere. This can only occur in the spherical approximation for asteroids with mass ratios $q \lesssim 0.2$, because only for these systems is the critical spin rate high enough that the free energy is positive and disruption trajectories are available (Scheeres 2007a). As shown in Figure 1, the observed asteroid pairs obey the relationship between the rotation rate of the larger, primary member of the pair and the mass ratio predicted by considerations of the free energy of a YORPinduced rotational fission event; this is clearly the primary mechanism for the formation of asteroid pairs (Pravec et al. 2010). We briefly review the theory here and then expand the theory to consider the roles of strength and secondary fission on the relationship 


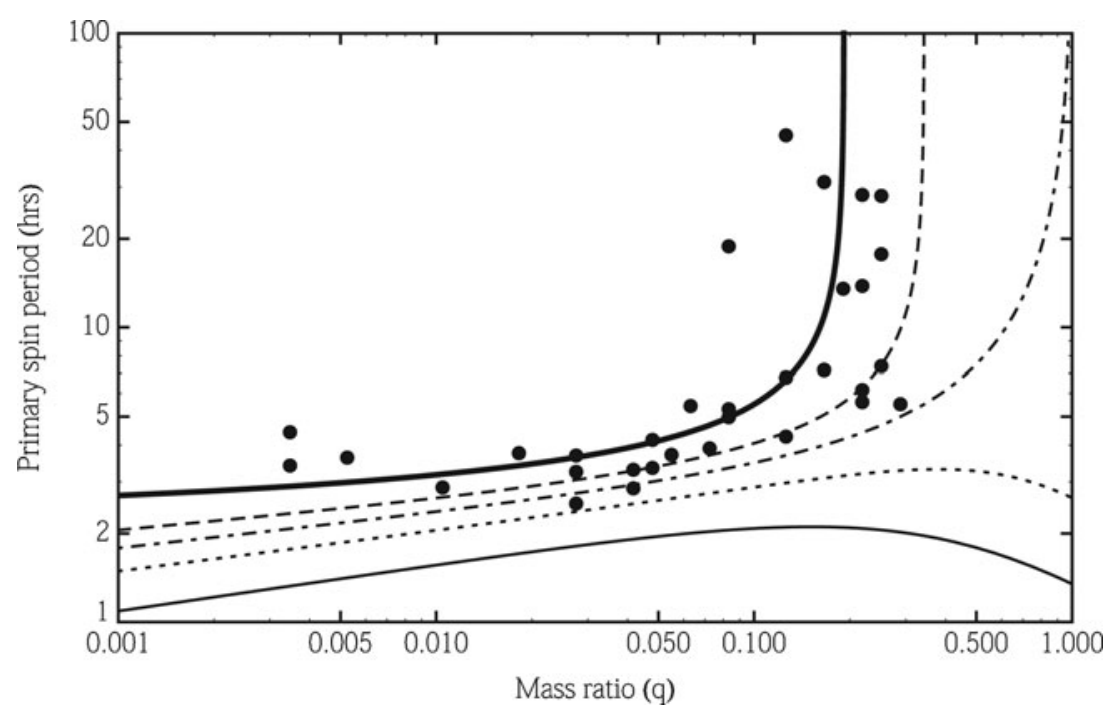

Figure 1. The final spin period of the larger, primary component of an asteroid pair after rotational fission as a function of mass ratio. The dots are measured spin periods and mass ratios of observed asteroid pairs from Pravec et al. (2010). The lines show the spin period-mass ratio relationship for a planar, two sphere approximation for a rotationally fissioned progenitor with different strengths: strengthless $\left(\omega_{t}=0\right.$; bold, solid), while the others are $\omega_{t}=0.243 \omega_{d}$ (dashed), $\omega_{t}=0.324 \omega_{d}$ (dot-dashed), $\omega_{t}=0.432 \omega_{d}$ (dotted), and $\omega_{t}=0.648 \omega_{d}$ (thin, solid). The critical tensile strength spin rate is $\omega_{t}=\sqrt{3 \sigma_{c} /\left(4 \pi \rho R^{2}\right)}$, where the absolute tensile strength is $\sigma_{c}=\sigma_{c, 0} \sqrt{R_{0} / R}$. With the caveat that these parameters $\left(\sigma_{c, 0}=125 \mathrm{~Pa}\right.$ and $\left.R_{0}=165\right)$ are poorly characterized as discussed in the text, the lines representing bodies with strength correspond to a range of sizes: $500 \mathrm{~m}, 400 \mathrm{~m}, 320 \mathrm{~m}$ and $230 \mathrm{~m}$, respectively.

between mass ratio and primary spin rate. These may be the principal mechanisms for the production of outliers to the theory as presented in Pravec et al. (2010).

\subsection{Asteroid pair formation directly from a strengthless asteroid}

Within the closed system of a binary formed after YORP-induced rotational fission, the free energy must be conserved. If the two components scatter each other onto a disruption trajectory due to higher-order non-Keplerian gravitational terms, i.e. spin-orbit coupling, then the free energy available to the spin states of each component is reduced since some energy is permanently lost to escape their mutual gravitational potential. Also, the ratio of the rotational energy in the secondary to the primary is $\propto q^{5 / 3} \omega_{s} / \omega_{p}$, which means for similar spin rates and low mass ratios $q \lesssim 0.2$, the free energy stored in the secondary is relatively insignificant, so we assert as a first-order approximation that the secondary is rotating at the critical spin rate. Again, we use a planar two-sphere approximation, however it must be recognized that some asphericity is necessary for the two components to have found a disruption trajectory from their initially binary state after rotational fission, although the level of asphericity needed is very low for the binary system to explore phase space and find an escape trajectory since the initial semi-major axis of the system is very small compared to the radius of the primary (Jacobson and Scheeres 2011a). Then, given these assumptions, the free energy of the disrupted system is only the energy in the two spin states:

$$
\frac{E_{d}}{E_{c}}=q_{3}\left(q_{d}^{2}+\frac{\omega_{p}^{2}}{\omega_{d}^{2}} q^{-5 / 3}\right) \quad q_{3}=2\left(q^{2}(1+q)\right)^{1 / 3}
$$


where $\omega_{p}$ is the spin rate of the primary. Setting this energy equal to the free energy available after fission, the final spin rate of the primary is: $\omega_{p} / \omega_{d}=q^{5 / 3}\left(\left(q_{1}-q_{3}\right) q_{d}^{2}-q_{2}\right) / q_{3}$. This spin rate as a rotation period is plotted (bold, solid line) as a function of mass ratio in Figure 1.

This simple relationship provides an easy to test hypothesis for the asteroid pair population, and the data fit the prediction well (Pravec et al. 2010), as shown. Some of the systems stray from the predicted relation, and Pravec et al. (2010) explored how changes in the spherical approximation may account for these discrepancies. In particular, asserting that the components have different shapes changes the initial free energy system since the critical spin rate for rotational fission is a function of the distance between the mass centers of the components. We can imagine at least two other modifications to the scenario above that may create asteroid pairs that do not follow the above relation.

\subsection{Asteroid pair formation directly from an asteroid with strength}

As discussed above, asteroids likely have significant tensile strength as their sizes decrease. If this is the case, then the free energy $E_{i}$ available to the binary system will be greater than in the case of only gravity, and using the same approximations as above for the free energy of the disrupted asteroid pair system, the final spin rate of the primary is:

$$
\frac{E_{i}}{E_{c}}=q_{1}\left(q_{d}^{2}+\frac{\omega_{t}^{2}}{\omega_{d}^{2}} q_{t}^{2}\right)-q_{2} \quad \frac{\omega_{p}}{\omega_{d}}=\sqrt{\frac{q^{5 / 3}\left(\left(q_{1}-q_{3}\right)\left(q_{d}^{2}+q_{t}^{2}\left(\frac{\omega_{t}}{\omega_{d}}\right)^{2}\right)-q_{2}\right)}{q_{3}}}
$$

where the strength of the progenitor asteroid is characterized by the ratio of the critical tensile strength spin limit to the critical gravitational spin rate $\omega_{t} / \omega_{d}$. When this ratio is zero, the strengthless solution is obtained, but as this ratio increases, the primary spin rate after disruption increases for a given mass ratio. Because the spin rate necessary to fission the progenitor increases with $\omega_{t}$, increasingly higher mass ratio systems have positive free energies and can disrupt. For instance, the minimum necessary strength of the progenitor asteroid in order for it to spin fission in half (i.e. $q=1$ ) is $\omega_{t} / \omega_{d} \approx 0.324$. Given the nominal asteroid strength relationship between critical tensile strength spin limit and size as determined above, this occurs for an asteroid with a radius of 400 m. A larger asteroid is unlikely to fission in half and form an unbound asteroid pair, while a smaller asteroid may fission in half and even have a rapidly rotating primary. In general, asteroids may span a wide variety of critical strengths due to variations in size and strength laws, and so a number of different $\omega_{t} / \omega_{d}$ ratios are plotted in Figure 1.

In summary, if the progenitor asteroid has strength, then it is possible for the primary to rotate at low periods even when the mass ratio is relatively high. Moreover, the critical mass ratio is no longer 0.2 , but a function of the ratio $\left(\omega_{t} / \omega_{d}\right)$, which is directly related to the critical strength and therefore, the size of the progenitor asteroid.

\subsection{Asteroid pair formation after a secondary fission event}

The asteroid pair production process was found to be incredibly efficient, and secondary fission was proposed as mechanism to stabilize rotationally fissioned asteroids to create long-lasting binary systems (Jacobson and Scheeres 2011a). Secondary fission occurs when the smaller, secondary binary member is torqued due to spin-orbit couping to a critical spin rate and thus, the secondary itself undergoes rotational fission. For simplicity, we only consider strengthless asteroids, so the initial free energy is the same as in that case. Now however, there is a binary system paired with the disrupted tertiary member, so the free energy includes terms for the spin states of all three bodies, their relative 


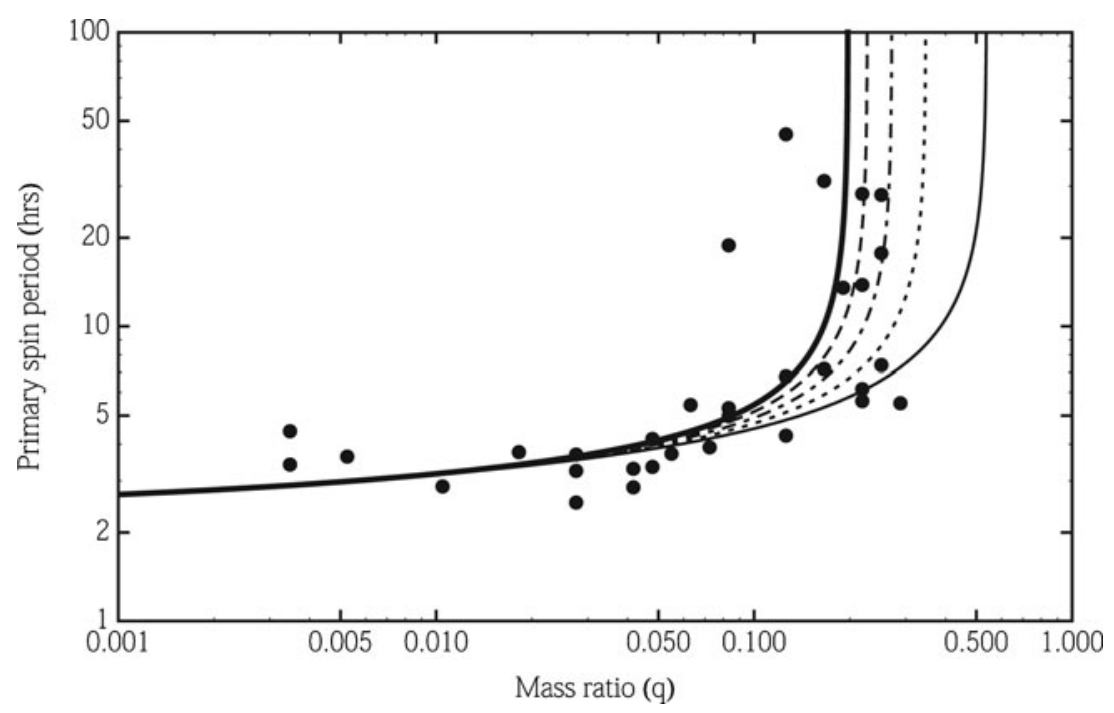

Figure 2. Similar to Figure 1, the final spin period of the larger, primary component of an asteroid pair after both rotational fission and secondary fission as a function of mass ratio $q$. The dots are measured spin periods and mass ratios of observed asteroid pairs from Pravec et al. (2010). The lines show a planar, three sphere approximation for a paired binary system with semi-major axes of infinite (bold, solid), 6 (dashed), 3 (dot-dashed), 2 (dotted) and 1.5 (thin, solid) primary radii assuming an equal mass secondary fission event, so a mass ratio $p=1$.

translational energies, and the mutual potential energy of the binary:

$$
\frac{E_{b}}{E_{c}}=q_{3}\left(p_{1}+\frac{\omega_{p}^{2}}{\omega_{d}^{2}} q^{-5 / 3}\right)-q_{2} \frac{1+q^{1 / 3}}{2 a(1+p)} \quad p_{1}=\frac{1-\left(1-p^{1 / 3}\right)\left(1+p^{2 / 3}\right) p^{1 / 3}}{\left(1+p^{1 / 3}\right)^{2}(1+p)^{2 / 3}}
$$

where $p$ is the mass ratio of the smaller, tertiary component to the larger, secondary component after secondary fission. The final spin rate of the primary is then a function of both relevant mass ratios $q$ and $p$ as well as the semi-major axis $a$ measured in primary radii $R_{p}$ of the bound system:

$$
\frac{\omega_{p}}{\omega_{d}}=\sqrt{\frac{q^{5 / 3}\left(\left(1+q^{1 / 3}\right) q_{2}-2(1+p)\left(q_{2}-q_{1} q_{d}^{2}+q_{3} p_{1}\right) a\right)}{2 q_{3}(1+p) a}}
$$

This relationship between primary spin rate and the mass ratio of the bound system to the unbound pair member is shown in Figure 2. While there is a dependence on the semimajor axis of the bound system, it's remarkable how unchanged the expected spin period of the primary in a paired binary compared to a lone primary. This is important, because the secondary fission hypothesis suggests that most low mass ratio binaries formed via this process (Jacobson and Scheeres 2011a). Indeed, two such systems have already been discovered: 3749 Balam is a triple system with an associated pair member (Vokrouhlický 2009) and 8306 Shoko is a binary system with an associated pair member (Pravec et al. 2013). The creation of these binary systems simultaneously with their pair members means that binary evolution can be tied to a well estimated timescale. Thus, this enables a powerful technique to learn about tidal parameters and asteroid geophysics. 


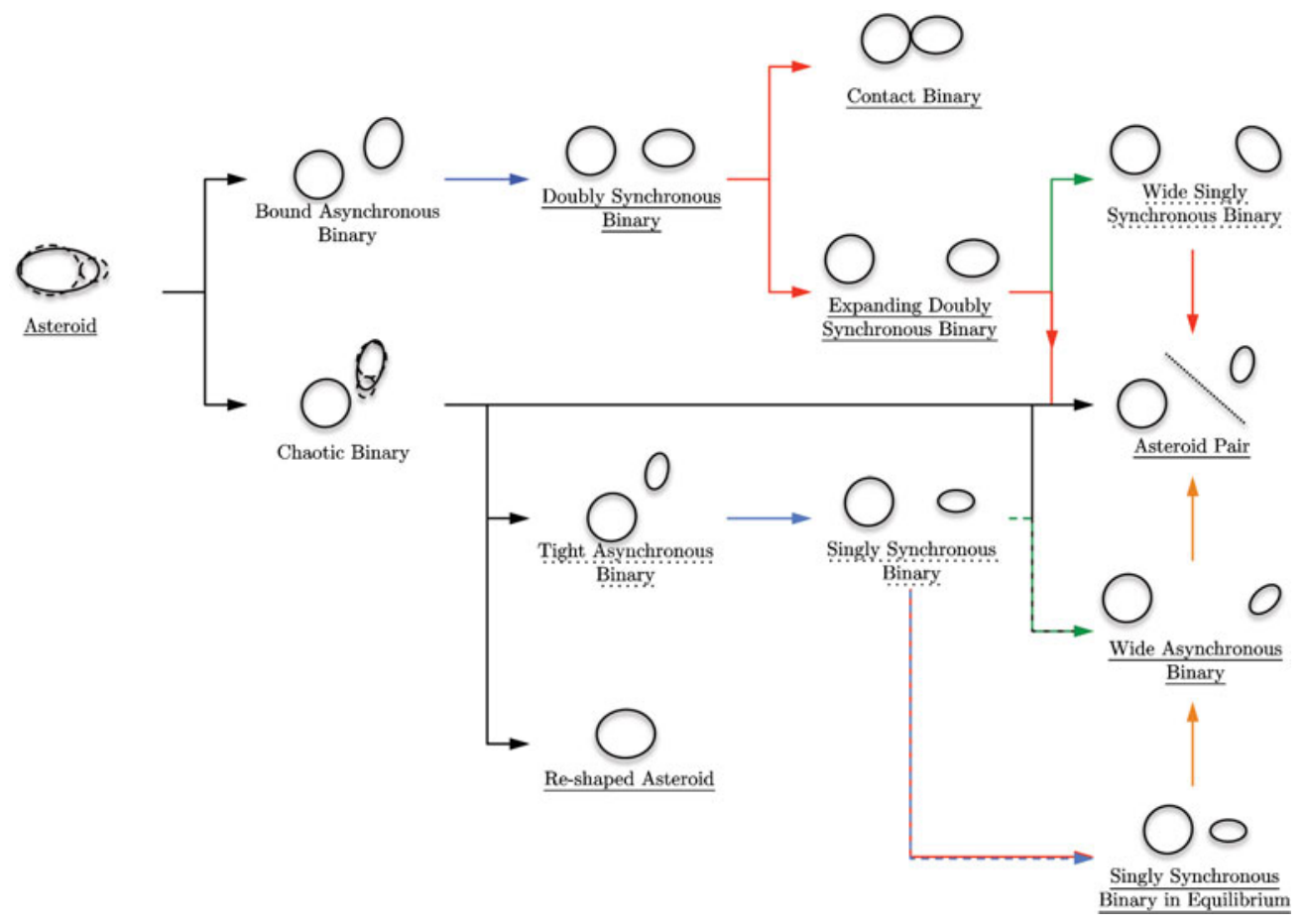

Figure 3. Flowchart depicting the evolution of an asteroid after a YORP-induced rotational fission event (reproduced from Jacobson (2014)). Arrows indicate direction of evolution between cartoons and labels of the different evolutionary states. Solid and dashed underlines indicate long- and short-term stability, respectively. Colors indicate the dominant evolutionary process: black (purely dynamical), blue (tidal), red (BYORP effect), green (YORP effect), and yellow (planetary flyby).

\section{Alternative asteroid pair formation mechanisms}

The binary evolution model that has been developed to tie together the observed binary systems is reviewed in detail in Jacobson (2014), and it is best summarized by the flowchart in Figure 3. If after YORP-induced rotational fission the asteroid is bound (negative free energy), then it follows the upper path, whereas if the asteroid is unbound (positive free energy) it follows the lower path. Asteroid pairs formed directly from rotational fission or secondary fission events are indicated by the middle path. This schematic shows four other paths to asteroid pair formation, which we discuss in turn below after introducing mutual body tides and the BYORP effect.

Mutual body tides are gravitational torques that arise from the delayed response of each binary component to the changing gravitational field of the system. If the bodies were inviscid fluids that reacted instantly to gravity, then these tides would be nonexistent. However, real asteroids react viscously to tides dissipating energy in the form of heat and tidally torquing the orbit (Efroimsky 2015). For the binary systems created by YORP-induced rotational fission, these tidal torques typically expand the semi-major axis and damp the eccentricity of the mutual orbit (Goldreich and Sari 2009).

The binary YORP (BYORP) effect is similar to the YORP effect, but instead of an averaged thermal radiation torque on the spin state, it is an average thermal radiation torque on the mutual orbit. Typically this effect averages to zero, since the relative orientation of the binary components is effectively random with respect to the mutual orbit, but when either or both of the binary components is in a spin-orbit resonance, 
then the BYORP effect becomes non-negligible (Ćuk and Burns 2005). The direction of this torque on the orbit is dependent on the shape and orientation of the bodies in the spin-orbit resonance, and so it can either contract or expand the orbit (McMahon and Scheeres 2010b).

\subsection{Asteroid pair formation from singly synchronous mutual orbit expansion}

Binary asteroids formed from YORP-induced rotational fission with low mass ratios typically tidally synchronize only the secondary since the tidal synchronization timescales are so different (Goldreich and Sari 2009; Jacobson and Scheeres 2011a). Once synchronized, the secondary begins to evolved due to the BYORP effect (Ćuk 2007; McMahon and Scheeres 2010a). If it evolves inward, it likely reaches a tidal-BYORP equilibrium, which matches the properties of most observed small binary asteroid systems (Jacobson and Scheeres 2011b; Scheirich et al. 2015). However, if the BYORP effect expands the mutual orbit, then it may expand to the Hill radius. Given estimates of the strength of the BYORP effect (McMahon and Scheeres 2010a, 2012), semi-major axis growth may take $\sim 10^{5}-10^{7}$ years before it reaches the Hill radius of the system. Once it reaches the Hill radius, the binary will disrupt.

Asteroid pairs created via this route take significantly longer to form after the rotational fission event than asteroid pairs created directly from rotational fission, which may form only $\sim 0-10^{3}$ years after the rotational fission event. This has significant implications for interpreting the dynamical ages of asteroid pairs determined from backwards integration of the heliocentric orbits. Furthermore, the secondaries of these asteroid pairs have been tidally decelerated before the system is disrupted. This is very different than the secondaries of asteroid pairs formed directly from rotational fission are as likely to still be rapidly rotating as not since the spin-orbit coupling during the temporary binary phase after YORP-induced rotational fission is best approximated by a random walk (Jacobson and Scheeres 2011a).

\subsection{Asteroid pair formation from doubly synchronous mutual orbit expansion}

Binary asteroids formed from YORP-induced rotational fission with negative free energy typically have high mass ratios and so tidally synchronize both binary components. Tidal synchronization timescales for small $(R<10 \mathrm{~km})$ asteroids in this configuration are typically less than or similar to near-Earth asteroid lifetimes and so significantly shorter than main belt collisional lifetimes (Goldreich and Sari 2009; Jacobson and Scheeres 2011a). Once tidally locked, these binaries undergo BYORP effect driven evolution. The direction of this mutual orbit evolution is dependent on the shapes and orientations of both binary members; it's possible that the torque on each member add in the same direction or subtract from each other, but it's unlikely that they perfectly cancel. Like above, semi-major axis growth may take $\sim 10^{5}-10^{7}$ years before the mutual orbit expands to the Hill radius of the system. Once it reaches the Hill radius, the binary disrupts.

Asteroid pairs created via this mechanism will have very different characteristics than those formed directly from YORP-induced rotational fission or after a secondary fission event. These asteroid pairs are likely to have high mass ratios and low spin rates. Furthermore, the dynamical age determined from backwards integrating the heliocentric orbits of the pair members, again like above, does not simply correspond with the date of the rotational fission events. 
3.3. Asteroid pair formation from mutual orbit expansion after de-synchronization of one member

The most complicated scenario envisioned for the formation of asteroid pairs occurs when both members of doubly synchronous binary asteroids have expansive BYORP torques and that during this expansion, once of the binary components desynchronizes and begins to circulate due to the YORP effect. This is very similar to the process that forms the wide asynchronous binary population (Jacobson et al. 2014b), but one of the components is still synchronous. Thus the BYORP effect continues to expand the system to the Hill radius and once it reaches the Hill radius, the binary disrupts.

Asteroid pairs created by this chain of events would appear strange. They would have a high mass ratio, but one could be rotating quite rapidly (it could be either the primary or the secondary) while the other rotates very slowly. Like the previous two mechanisms, the dynamical age determined from backwards integration of the asteroid pair is not indicative of the timing of the rotational fission event.

\subsection{Asteroid pair formation from planetary flybys}

The simplest formation mechanism for asteroid pairs can only occur when binary asteroids are on planet crossing orbits. Then it is quite possible for planetary flybys to disrupt these binaries (Fang and Margot 2012), however the perturbations from the very planet that caused the disruption may make the determination of an asteroid pair quite difficult. This process could occur to any binary asteroid system morphology, so the observed asteroid pair properties would span a large range.

\section{Conclusions}

In this proceedings, we have summarized the evidence that most asteroid pairs form either directly from YORP-induced rotational fission or subsequent secondary fission events. This is important because asteroid pair formation is then intimately tied to binary formation. Indeed, the discovery that some binary asteroids like 8306 Shoko and 3749 Balam have unbound pair members is likely to create stringent constraints on binary evolution in the near future. We have also summarized the different asteroid pair formation mechanisms due to the BYORP effect and planetary flybys. These asteroid pairs can appear very different but also similarly to those that formed directly following a rotational fission event. Importantly, the interpretation of the dynamical age of the asteroid pair system is very different.

\section{References}

Bottke, W. F., Vokrouhlický, D., Rubincam, D. P., \& Brož, M. (2002). The Effect of Yarkovsky Thermal Forces on the Dynamical Evolution of Asteroids and Meteoroids. Asteroids III, pages $395-408$.

Breiter, S., Michalska, H., Vokrouhlický, D., \& Borczyk, W. (2007). Radiation-induced torques on spheroids. Astronomy and Astrophysics, 471(1):345-353.

Ćuk, M. (2007). Formation and Destruction of Small Binary Asteroids. The Astrophysical Journal, 659(1):L57-L60.

Ćuk, M. \& Burns, J. A. (2005). Effects of thermal radiation on the dynamics of binary NEAs. ICARUS, 176(2):418-431.

Duddy, S. R., Lowry, S. C., Wolters, S. D., Christou, A., Weissman, P. R., Green, S. F., \& Rozitis, B. (2012). Physical and dynamical characterisation of the unbound asteroid pair 7343-154634. Astronomy and Astrophysics, 539:A36. 
Efroimsky, M. (2015). Tidal Evolution of Asteroidal Binaries. Ruled by Viscosity. Ignorant of Rigidity. The Astronomical Journal, 150(4):98.

Fang, J. \& Margot, J.-L. (2012). Binary Asteroid Encounters with Terrestrial Planets: Timescales and Effects. The Astronomical Journal, 143(1):25.

Goldreich, P. \& Sari, R. (2009). Tidal Evolution of Rubble Piles. The Astrophysical Journal, 691(1):54-60.

Golubov, O. \& Krugly, Y. N. (2012). Tangential Component of the YORP Effect. The Astrophysical Journal Letters, 752(1):L11.

Golubov, O., Scheeres, D. J., \& Krugly, Y. N. (2014). A Three-dimensional Model of Tangential YORP. The Astrophysical Journal, 794(1):22.

Harris, A. W. (1996). The Rotation Rates of Very Small Asteroids: Evidence for 'Rubble Pile' Structure. Abstracts of the Lunar and Planetary Science Conference, 27:493.

Hartmann, W. K. \& Larson, S. M. (1967). Angular momenta of planetary bodies. ICARUS, $7(1): 257-260$.

Hergenrother, C. W. \& Whiteley, R. J. (2011). A survey of small fast rotating asteroids among the near-Earth asteroid population. ICARUS, 214(1):194-209.

Hirabayashi, M. \& Scheeres, D. J. (2015). Stress and Failure Analysis of Rapidly Rotating Asteroid (29075) 1950 DA. The Astrophysical Journal Letters, 798(1):L8.

Hirabayashi, M., Scheeres, D. J., Sánchez, D. P., \& Gabriel, T. (2014). Constraints on the Physical Properties of Main Belt Comet P/2013 R3 from its Breakup Event. The Astrophysical Journal Letters, 789(1):L12.

Holsapple, K. A. (2001). Equilibrium Configurations of Solid Cohesionless Bodies. ICARUS, 154(2):432-448.

Jacobson, S. A. (2014). Small asteroid system evolution. Proceedings of the International Astronomical Union, 310:108-117.

Jacobson, S. A., Marzari, F., Rossi, A., Scheeres, D. J., \& Davis, D. R. (2014a). Effect of rotational disruption on the size-frequency distribution of the Main Belt asteroid population. Monthly Notices of the Royal Astronomical Society: Letters, page L15.

Jacobson, S. A. \& Scheeres, D. J. (2011a). Dynamics of rotationally fissioned asteroids: Source of observed small asteroid systems. ICARUS, 214(1):161-178.

Jacobson, S. A. \& Scheeres, D. J. (2011b). Long-term Stable Equilibria for Synchronous Binary Asteroids. The Astrophysical Journal Letters, 736(1):L19.

Jacobson, S. A., Scheeres, D. J., \& McMahon, J. W. (2014b). Formation of the Wide Asynchronous Binary Asteroid Population. The Astrophysical Journal, 780(1):60.

Kaasalainen, M., Ďurech, J., Warner, B. D., Krugly, Y. N., \& Gaftonyuk, N. M. (2007). Acceleration of the rotation of asteroid 1862 Apollo by radiation torques. Nature, 446(7):420-422.

Lowry, S. C., Fitzsimmons, A., Pravec, P., Vokrouhlický, D., Boehnhardt, H., Taylor, P. A., Margot, J.-L., Galad, A., Irwin, M., Irwin, J., \& Kusnirák, P. (2007). Direct Detection of the Asteroidal YORP Effect. Science, 316(5):272-.

McMahon, J. W. \& Scheeres, D. J. (2010a). Detailed prediction for the BYORP effect on binary near-Earth Asteroid (66391) 1999 KW4 and implications for the binary population. ICARUS, 209(2):494-509.

McMahon, J. W. \& Scheeres, D. J. (2010b). Secular orbit variation due to solar radiation effects: a detailed model for BYORP. Celestial Mechanics and Dynamical Astronomy, 106(3):261300 .

McMahon, J. W. \& Scheeres, D. J. (2012). Binary-YORP Coefficients for Known Asteroid Shapes. Bulletin of the American Astronomical Society, 44.

Moskovitz, N. A. (2012). Colors of dynamically associated asteroid pairs. ICARUS, 221(1):63-71.

Nesvorný, D. \& Vokrouhlický, D. (2007). Analytic Theory of the YORP Effect for Near-Spherical Objects. The Astronomical Journal, 134(5):1750.

Pravec, P. \& Harris, A. W. (2000). Fast and Slow Rotation of Asteroids. ICARUS, 148(1):12-20.

Pravec, P., Harris, A. W., \& Warner, B. D. (2007). NEA rotations and binaries. Near Earth Objects, 236:167-176.

Pravec, P., Kusnirák, P., Hornoch, K., Galad, A., Krugly, Y. N., Chiorny, V. G., Inasaridze, R. Y., Kvaratskhelia, O., Ayvazian, V., Parmonov, O., Pollock, J., Mottola, S., Oey, J., 
Pray, D., Žižka, J., Vraštil, J., Molotov, I. E., Reichart, D. E., Ivarsen, K. M., Haislip, J. B., \& Lacluyze, A. P. (2013). (8306) Shoko. IAU Circ., 9268:1.

Pravec, P. \& Vokrouhlický, D. (2009). Significance analysis of asteroid pairs. ICARUS, 204(2):580-588.

Pravec, P., Vokrouhlický, D., Polishook, D., Scheeres, D. J., Harris, A. W., Galad, A., Vaduvescu, O., Pozo, F., Barr, A., Longa, P., Vachier, F., Colas, F., Pray, D. P., Pollock, J., Reichart, D. E., Ivarsen, K. M., Haislip, J. B., Lacluyze, A. P., Kusnirák, P., Henych, T., Marchis, F., Macomber, B., Jacobson, S. A., Krugly, Y. N., Sergeev, A. V., \& Leroy, A. (2010). Formation of asteroid pairs by rotational fission. Nature, 466(7):1085-1088.

Rozitis, B., MacLennan, E., \& Emery, J. P. (2014). Cohesive forces prevent the rotational breakup of rubble-pile asteroid (29075) 1950 DA. Nature, 512(7):174-176.

Rubincam, D. P. (2000). Radiative Spin-up and Spin-down of Small Asteroids. ICARUS, 148(1):2-11.

Sánchez, D. P. \& Scheeres, D. J. (2014). The strength of regolith and rubble pile asteroids. Meteoritics $\&$ Planetary Science, 49(5):788-811.

Scheeres, D. J. (2007a). Rotational fission of contact binary asteroids. ICARUS, 189:370.

Scheeres, D. J. (2007b). The dynamical evolution of uniformly rotating asteroids subject to YORP. ICARUS, 188(2):430-450.

Scheeres, D. J. (2009). Stability of the planar full 2-body problem. Celestial Mechanics and Dynamical Astronomy, 104(1):103-128.

Scheirich, P., Pravec, P., Jacobson, S. A., Ďurech, J., Kusnirák, P., Hornoch, K., Mottola, S., Mommert, M., Hellmich, S., Pray, D., Polishook, D., Krugly, Y. N., Inasaridze, R. Y., Kvaratshelia, O. I., Ayvazian, V., Slyusarev, I., Pittichová, J., Jehin, E., Manfroid, J., Gillon, M., Galad, A., Pollock, J., Licandro, J., Alí-Lagoa, V., Brinsfield, J., \& Molotov, I. E. (2015). The binary near-Earth Asteroid (175706) 1996 FG3 - An observational constraint on its orbital evolution. ICARUS, 245:56-63.

Sharma, I. (2009). The equilibrium of rubble-pile satellites: The Darwin and Roche ellipsoids for gravitationally held granular aggregates. ICARUS, 200(2):636-654.

Taylor, P. A., Margot, J.-L., Vokrouhlický, D., Scheeres, D. J., Pravec, P., Lowry, S. C., Fitzsimmons, A., Nolan, M. C., Ostro, S. J., Benner, L. A. M., Giorgini, J. D., \& Magri, C. (2007). Spin Rate of Asteroid (54509) 2000 PH5 Increasing Due to the YORP Effect. Science, 316(5):274-.

Vokrouhlický, D. (2009). (3749) Balam: A Very Young Multiple Asteroid System. The Astrophysical Journal Letters, 706(1):L37-L40.

Vokrouhlický, D. \& Čapek, D. (2002). YORP-Induced Long-Term Evolution of the Spin State of Small Asteroids and Meteoroids: Rubincam's Approximation. ICARUS, 159(2):449-467.

Vokrouhlický, D. \& Nesvorný, D. (2008). Pairs of Asteroids Probably of a Common Origin. The Astronomical Journal, 136(1):280-290.

Vokrouhlický, D. \& Nesvorný, D. (2009). The Common Roots of Asteroids (6070) Rheinland and (54827) 2001 NQ8. The Astronomical Journal, 137(1):111-117.

Vokrouhlický, D., Nesvorný, D., \& Bottke, W. F. (2003). The vector alignments of asteroid spins by thermal torques. Nature, 425(6):147-151.

Walsh, K. J., Richardson, D. C., \& Michel, P. (2008). Rotational breakup as the origin of small binary asteroids. Nature, 454(7):188-191.

Wolters, S. D., Weissman, P. R., Christou, A., Duddy, S. R., \& Lowry, S. C. (2014). Spectral similarity of unbound asteroid pairs. Monthly Notices of the Royal Astronomical Society, 439(3):3085-3093. 